\section{Implementación de una unidad de artritis reumatoide temprana en pacientes chilenos derivados desde centros de atención primaria de salud}

\author{
FRANCISCO ESPINOZA ${ }^{1}$, GUSTAVO MONCKEBERG ${ }^{1}$, \\ ISABEL HASSI $^{1}$, ALEJANDRA QUEIROLO ${ }^{1}$, FERNANDO CHICAO $^{1}$, \\ XIMENA SANDOVAL ${ }^{2}$, EVELYN JORQUERA ${ }^{2}$, ALEJANDRO BADILLA ${ }^{1}$
}

\section{Implementation of an early rheumatoid athritis unit for the early recognition and treatment of the disease}

Background: Early recognition of rheumatoid arthritis $(R A)$ provides clinical benefits in terms of remission induction, reduced disease progression, and eventually treatment free remission. Aim: To describe the setting of a Unit devoted exclusively to the recognition and treatment of early $R A$ in patients referred from primary healthcare centers (PHC) in Chile. Materials and Methods: Patients were referred from nine participating PHC from 2014 through 2016. PHC physicians received a formal training to enhance criteria recognition and program adherence. Mandatory referral criteria were an age above 17 years, and arthralgia of less than 1-year duration, plus at least one of the following: morning stiffness of more than 30 minutes, swelling involving more than 3 joints for more than 1 month, a positive squeeze test or abnormal inflammatory serum markers. Results: One hundred twenty patients aged $45 \pm 12$ years (90\% women) were assessed at the early rheumatoid arthritis unit. Median time to referral from PHC to the Unit was 14.6 days. The median duration of symptoms for the overall sample of patients was 10.8 months. RA was identified in 43 patients (36\%), with a delay between onset of symptoms and diagnosis of 8.3 months. Regarding the performance of referral criteria, the most sensitive was morning stiffness ( $80 \%$, sensitivity 95\% confidence intervals (CI) 64-89\%) and synovitis was the most specific (specificity 83\%, 95\% CI 72-90\%). The positive predictive value of the three clinical criteria altogether was $68.1 \%$ (95\% CI 47-83\%). Conclusions: Institution of an early RA unit was feasible within the Chilean healthcare system enabling the identification of early RA in one-third of patients.

(Rev Med Chile 2018; 146: 39-45)

Key words: Arthritis, Rheumatoid; Diagnosis; Therapeutics; Time-toTreatment.

\author{
'Escuela de Medicina, Universidad \\ de los Andes. Santiago, Chile. \\ ${ }^{2}$ Centro de Referencia de Salud \\ Cordillera, Servicio de Salud \\ Metropolitano Oriente, Ministerio \\ de Salud. Santiago, Chile. \\ Los autores declaran no tener \\ conflictos de interés. \\ Trabajo no recibió \\ financiamiento.
}

Recibido el 11 de mayo de 2017, aceptado el 9 de enero de 2018.

Correspondencia a:

Dr. Francisco Espinoza V.

Unidad de Reumatología,

Departamento de Medicina Interna, Escuela de Medicina,

Universidad de los Andes.

Avenida Álvaro del Portillo

12455. Las Condes.

Santiago, Chile.

fespinoza@uandes.cl
L a artritis reumatoide (AR) es una enfermedad autoinmune sistémica que ocasiona grados variables de discapacidad y aumento de la morbi-mortalidad. Afecta al 0,5-1\% de la población mundial y su elevada carga de enfermedad ha hecho que en Chile sea incorporada dentro de las patologías con garantías explícitas en salud $(\mathrm{GES})^{1,2}$. Una de cada tres personas con AR tiene algún grado de limitación física y hasta $20 \%$ abandona la vida laboral dentro de los primeros 5 años de enfermedad ${ }^{3}$.

La implementación de una estrategia de tra- 
tamiento por objetivos ("treat-to-target", T2T) ${ }^{4}$ ha puesto de relieve la necesidad de lograr una remisión prolongada que permita maximizar la calidad de vida al normalizar la función articular y prevenir secuelas. Concretar este objetivo depende, en parte, de implementar herramientas que favorezcan un diagnóstico y tratamiento precoz. El tiempo de evolución de la enfermedad es uno de los factores predictores de éxito más relevantes, independiente del fármaco prescrito ${ }^{5}$. En este sentido, el concepto de "ventana de oportunidad" ha mostrado los beneficios de una intervención precoz, con la detención de la progresión y la obtención de tasas de remisión libre de tratamiento superiores $^{6-8}$.

Las unidades de artritis temprana permiten cristalizar los principios del cuidado moderno de la AR, considerando una estrategia de diagnóstico y tratamiento intensiva. El objetivo de la presente comunicación es describir la implementación de una unidad dedicada a la AR temprana a partir de pacientes chilenos de atención primaria de salud.

\section{Pacientes y Métodos}

\section{Pacientes}

La unidad de AR temprana (UART) fue establecida en noviembre de 2014. Para su implementación se destinó un policlínico exclusivo en el Centro de Referencia de Salud (CRS) Cordillera perteneciente al Servicio de Salud Metropolitano Oriente en Santiago de Chile. La UART se conformó con un equipo de tres reumatólogos y una enfermera. El estudio se ajusta a los principios éticos de la Declaración de Helsinski exigida para un estudio de investigación en humanos.

El piloto consideró a los beneficiarios de las comunas de Macul y Peñalolén, estimados en 323.993 habitantes, a través de sus centros de salud familiar (CESFAM): Félix de Amesti, Padre Alberto Hurtado, Santa Julia, Cardenal Silva Henríquez, Carol Urzúa, La Faena, Lo Hermida, Padre Gerardo Whelan y San Luis. Se elaboraron criterios de derivación, entre los cuales se encuentran dos mandatorios (edad $>17$ años y menos de 12 meses de síntomas articulares), asociado a la presencia de al menos uno de los criterios clínicos de la Tabla 1. Los exámenes de laboratorio, incluyendo técnicas de laboratorio autoinmune o de imágenes fueron optativos con el objeto de no retardar la derivación.

La capacitación de los médicos de atención primaria se realizó en dos fases: En una primera instancia se organizó una reunión teórica-práctica con seis pacientes portadores de AR activa para que los profesionales tuvieran la posibilidad de reconocer y aplicar los criterios clínicos de derivación. En una segunda etapa, se reforzaron los contenidos con visitas programadas de los reumatólogos de la UART.

La derivación se realizó mediante interconsultas en línea dirigidas a la UART. Una vez visadas, los pacientes eran citados a una primera consulta de tamizaje donde eran vistos por el grupo de reumatólogos. A los pacientes con sospecha clínica de artritis se les solicitaba un estudio complementario basado en exámenes de laboratorio e imágenes. Si la AR era confirmada el paciente permanecía en la UART. De presentarse un diagnóstico alternativo que requiriese atención por especialista el paciente era derivado al policlínico de Reumatología y, finalmente, en ausencia de enfermedad reumatológica el paciente era derivado a su CESFAM. Todos los pacientes derivados a la UART se registraron en una base de datos electrónica confidencial.

\section{Variables clínicas y de laboratorio}

En la primera consulta de tamizaje fueron registradas las siguientes variables: sexo, edad, comorbilidades, escolaridad, medicamentos, tipo de dolor articular (mecánico o inflamatorio), distribución del dolor (mono, oligo o poliarticular), presencia de rigidez matinal, examen articular completo, compromiso extra-articular. En aquellos pacientes con sospecha de AR temprana, se complementó el estudio con laboratorio general e inmunológico (incluyendo al menos factor reumatoideo, anticuerpo anti-péptido cíclico citrulinado y anticuerpo antinucleares) e imagenología (radiografía de manos-pies y en casos específicos ecografía articular). Se utilizaron herramientas validadas de seguimiento como el índice compuesto DAS-28 (VHS) y de desempeño HAQ.

\section{Análisis estadístico}

Se realizó una descripción de la muestra usando promedios, medias y/o rangos para las variables cuantitativas y frecuencias para las variables cualitativas. La determinación de la sensibilidad (S), especificidad (E) y valor predictivo positivo (VPP) 
fue realizado mediante un análisis de contingencia utilizando el test exacto de Fisher. Para estimar la concordancia inter-observador se utilizó el coeficiente de Kappa. Los resultados se expresan con intervalos de confianza (IC) de 95\%. Los análisis fueron realizados mediante el programa Prism 7.0 (2016, GraphPad Software, Inc.) y para el cálculo de Kappa se utilizó la plataforma online http:// vassarstats.net/kappa.html

\section{Resultados}

\section{Características generales de la muestra}

Durante los últimos 24 meses fueron atendidos 120 pacientes. El tiempo de latencia entre la derivación desde atención primaria hasta la consulta de tamizaje fue de 14,6 $\pm 12,6$ días. El 90\% de los pacientes eran mujeres y el promedio de edad fue de $45 \pm 12$ años. La duración promedio de los síntomas fue de 10,8 \pm 9,6 meses. El compromiso poliarticular fue el más frecuente (86\%) (Tabla 2).

El diagnóstico de AR temprana fue realizado en $33 \%$ de los pacientes derivados. El segundo diagnóstico en frecuencia fue artrosis (18\%), seguido por fibromialgia (14\%). En un tercio de los pacientes otra patología fue diagnosticada. Este grupo se compone de AR establecida (7\%), síndrome de túnel carpiano (7\%), artritis indiferenciada (5\%), artritis por cristales (5\%), síndrome de sjoegren (3\%), lupus (2\%), espondiloartropatía axial (1\%), artritis psoriática (1\%) y otros reumatismos de partes blandas (4\%) (Figura 1).

\section{Rendimiento de los criterios de derivación}

El porcentaje de cumplimiento de cada criterio en orden descendente fue: a) mayores de 17 años: $100 \%$; b) ausencia de corticosteroides: $77 \%$; c) artralgia menor a 1 año: $73 \%$; d) rigidez matinal: 51\%; e) compresión sensible de metacarpo-falángicas y/o metatarsofalángicas: 36\%; f) sinovitis: $35 \%$ y g) elevación de parámetros inflamatorios (PCR y/o VHS): 35\%.

Para evaluar si la capacitación a los médicos de atención primaria fue efectiva en lograr un reconocimiento adecuado de los criterios de derivación, se analizó la concordancia inter-observador entre el médico general y el reumatólogo. La rigidez matinal obtuvo un grado de concordancia casi perfecto con un kappa de 0,88 (IC 95\% 0,79, 0,96). La sinovitis obtuvo un grado de concordancia moderado con un kappa de 0,53 (IC 95\% 0,38, $0,69)$ y el squeeze test fue el más bajo de los tres, obteniendo un grado de concordancia pobre de 0,16 (IC 95\% 0,01, 0,33).

Respecto al rendimiento de los criterios clíni-

\section{Tabla 1. Criterios de derivación a la Unidad de Artritis Reumatoide Temprana (UART)}

\section{Criterios obligatorios*}

I. Pacientes mayores de 17 años

II. Dolor articular menor a 1 año de evolución

\section{Criterios clínicos adicionales**}

a. Rigidez matinal de manos mayor a 30 minutos

b. Inflamación articular de más de 3 articulaciones por más de 4 semanas

c. Dolor a la compresión de metacarpofalángicas y/o metatarsofalángicas (squeeze test positivo)

d. Elevación de marcadores inflamatorios $(\mathrm{VHS} \text { y/o PCR })^{* * *}$

e. Sin uso de corticoides

*Los dos criterios deben estar presentes. ${ }^{* *} \mathrm{Al}$ menos un criterio debe estar presente. ${ }^{* * *}$ No era necesario derivar a un paciente con exámenes de laboratorio para no retardar evaluación en UART.

\section{Tabla 2. Características demográficas y clínicas de los pacientes durante la primera atención en la UART}

\begin{tabular}{|lc|}
\hline Variables generales & \\
Número de pacientes ( $\mathrm{n})$ & 120 \\
Sexo femenino, $\mathrm{n},(\%)$ & $99(90)$ \\
Edad (años, DS) & $45 \pm 12$ \\
Latencia de atención (días, DS) & $14,6 \pm 12,6$ \\
Variables clínicas & \\
Evolución de síntomas (meses, DS) & $10,8 \pm 9,6$ \\
Tipo de presentación articular (\%) & \\
$\quad$ Monoarticular & 3 \\
$\quad$ Oligoarticular & 4 \\
$\quad$ Poliarticular & 86 \\
$\quad$ Axial & 1 \\
$\quad$ Otro & 6 \\
Elevación de parámetros inflamatorios, n (\%) & $39(35)$ \\
Factor reumatoideo positivo, n (\%) & 35 (31) \\
Uso activo de corticosteroide oral (\%) & 23 \\
\hline
\end{tabular}




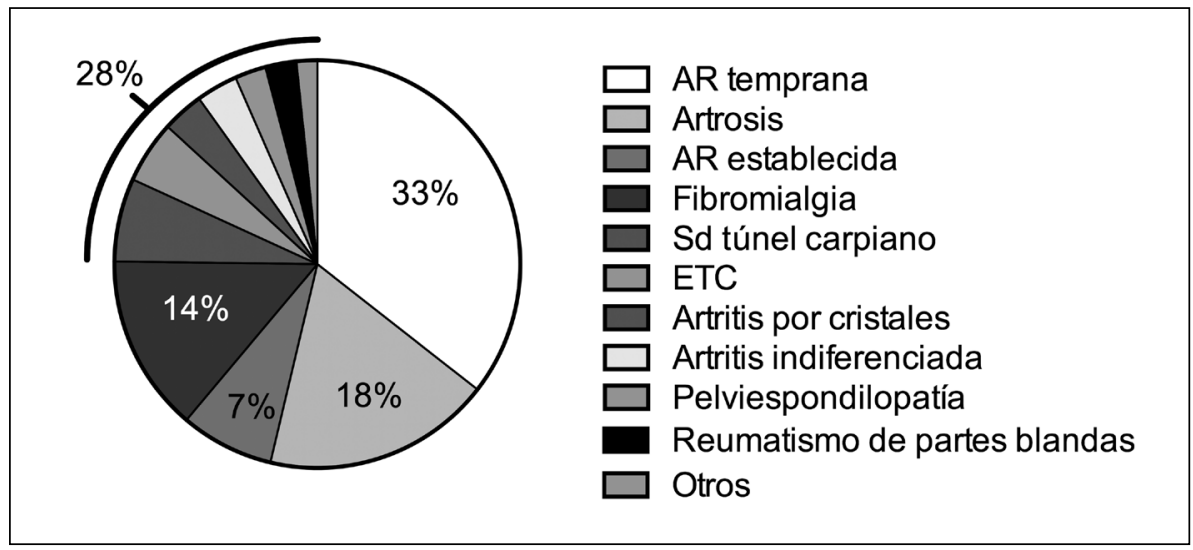

Figura 1.

cos en el diagnóstico de AR, el más sensible fue la rigidez matinal $(80 \%)$ y el más específico fue la sinovitis $(83 \%)$. El valor predictivo positivo del conjunto de los criterios clínicos fue de $68,1 \%$ (Tabla 3).

\section{Características clínicas de la muestra con artritis temprana}

De los individuos examinados en la UART, 43 cumplían criterios los criterios ACR/EULAR 2010 para AR. En el 95\% de los pacientes la conformidad de los criterios se completó dentro de los primeros 3 meses de seguimiento. Lo anterior representa al 33\% de la muestra total de pacientes. El 91\% de los pacientes con AR era de sexo femenino con un promedio de 47 años. En este grupo la duración promedio de los síntomas era de 8,3 meses. El compromiso articular de la mano fue el más frecuente. El 71\% de los pacientes tenían una AR seropositiva (FR 63,8\% y anti-CCP 67,2\%). Similar porcentaje tenían elevación de parámetros inflamatorios $(60 \%)$. La evaluación radiológica basal en la UART, mediante radiografía de manos y pies en proyección anteroposterior y oblicua, no reveló daño erosivo.

Los pacientes tenían una actividad moderada de la AR con un DAS 28-VHS inicial de 4,37 (rango, 2,17-7,32). El 20\% de los pacientes tenían algún compromiso extraarticular, siendo los más frecuentes el síndrome seco (14\%) y el compromiso pulmonar intersticial sospechado en la radiología y confirmado en la tomografía computada pulmonar $(7,3 \%)$. Respecto a la presencia de comorbilidades significativas en el seguimiento y actividad de la AR, se objetivó que $25 \%$ de los pacientes eran fumadores activos al momento de la inclusión y al menos $65 \%$ de los pacientes tenían algún factor de riesgo cardiovascular presente al momento del ingreso a la UAT: hipertensión arterial $37 \%$, dislipidemia $20 \%$ y diabetes mellitus 14\% (Tabla 4).

Respecto a la terapia, el tiempo promedio de inicio de un FARME fue de $31 \pm 23$ días desde la consulta de tamizaje. El fármaco más comunmente usado fue metotrexato (92\%). El uso de prednisona fue indicado en $80 \%$ de los casos a una dosis

Tabla 3. Sensibilidad y especificidad de los criterios de sospecha de compromiso articular inflamatorio, solicitados a los médicos de atención primaria para derivar un paciente a la UART

\begin{tabular}{|lcccc|}
\hline Criterio & $\begin{array}{c}\text { Sensibilidad } \\
(\mathbf{I C ~ 9 5 \% )}\end{array}$ & $\begin{array}{c}\text { Especificidad } \\
\text { (IC 95\%) }\end{array}$ & LR & P \\
\hline a. Rigidez matinal mayor a 30 minutos & $0,8(0,64,0,89)$ & $0,65(0,52,0,75)$ & 2,26 & 0,0001 \\
\hline b. Squeeze test & $0,51(0,35,0,67)$ & $0,72(0,6,0,81)$ & 1,85 & 0,02 \\
\hline c. Sinovitis $>3$ articulaciones $>$ 4 sem & $0,54(0,38,0,69)$ & $0,83(0,72,0,9)$ & 3,2 & 0,0002 \\
\hline Global $(A+B+C)$ & $0,42(0,27,0,59)$ & $0,95(0,83,0,98)$ & 4,98 & 0,006 \\
\hline
\end{tabular}


Tabla 4. Características clínicas basales de los pacientes en los cuales finalmente se hizo el diagnóstico de AR temprana

\begin{tabular}{|lc|}
\hline Variables clínicas & \\
Número de pacientes (n) & 41 \\
Sexo femenino (\%) & 91,8 \\
Edad, años (DS) & $47,3 \pm 13,2$ \\
Duración de los síntomas, meses (DS) & $8,3 \pm 6,4$ \\
Sitio articular (\%) & \\
$\quad$ MCFs, IFPs & 100 \\
$\quad$ Muñeca & 60,1 \\
$\quad$ Hombro/Codo & 31,5 \\
$\quad$ Cadera & 3 \\
$\quad$ Rodilla & 29,4 \\
$\quad$ Pie/Tobillo & 34,5 \\
$\quad$ Columna cervical & 0 \\
$\quad$ Temporo-mandibular & 0 \\
DAS-28 VHS, basal (rango) & 4,37 \\
Presencia de factor reumatoideo positivo (\%) & 63,8 \\
Presencia de anti-CCP positivo (\%) & 67,2 \\
Presencia de erosiones al diagnóstico (\%) & 0 \\
Sd. Inflamatorio biológico al diagnóstico (\%) & 60,4 \\
Compromiso extra-articular al diagnóstico (\%) & 20,2 \\
Comorbilidad & \\
Tabaquismo & 25,7 \\
Hipertensión arterial & 37,1 \\
Diabetes mellitus & 14,2 \\
Dislipidemia & 20 \\
Cardiopatía coronaria & 0 \\
\hline
\end{tabular}

media de inicio de $15 \mathrm{mg} /$ día (rango, 5-30 mg/día). La combinación de FARMEs más frecuentemente usada como estrategia inicial fue metotrexato + hidroxicloroquina en $14 \%$ de los pacientes.

\section{Discusión}

El objetivo del artículo es describir la metodología utilizada para implementar una unidad de artritis reumatoide temprana. Se describen los resultados obtenidos en términos de gestión y las características clínicas basales de la muestra. El presente artículo no considera un análisis prospectivo de la cohorte de pacientes con AR temprana.

La oportunidad para mejorar el cuidado, optimizar el pronóstico y disminuir los costos sanitarios y de morbilidad en AR, se relaciona con el factor tiempo. Un modelo sanitario que gestione diagnósticos tempranos con la consecuente introducción precoz de la terapia permite obtener beneficios entre los cuales se cuentan el reducir significativamente la progresión y el daño crónico, interrumpir el daño articular y mejorar las tasas de remisión libre de drogas ${ }^{9,10}$. Pacientes con una intervención terapéutica temprana tienen $33 \%$ menos de progresión radiológica comparado con aquellos que recibieron tratamiento "tardíamente", incluso cuando se considera a individuos con menos de dos años de enfermedad ${ }^{6}$. Van Nies et al, han demostrado recientemente que las posibilidades de alcanzar la remisión libre de drogas disminuye en $1 \%$ por cada semana que se retrasa la introducción de una terapia activa con FARMEs ${ }^{11}$. Resultados del registro DREAM comprueban que una estrategia terapéutica agresiva es adicionalmente costo-efectiva ${ }^{12}$.

En nuestra experiencia, se solicitó la derivación de pacientes con menos de 12 meses de duración de los síntomas. Es evidente que los plazos para definir una AR temprana se han ido modificando en el tiempo. Ensayos clínicos como el estudio $\mathrm{BeSt}^{13}$ o el estudio TEAR ${ }^{14}$, hace aproximadamente una década atrás, definieron AR temprana con una duración de síntomas de 2 y 3 años respectivamente. Registros y cohortes estadounidenses de AR temprana como ERATER ${ }^{15}$ o CLEAR ${ }^{16}$ establecidos entre el 2000 y 2005, incluyeron pacientes con igual tiempo de evolución. Cohortes más recientes, como SONORA ${ }^{17}$, han considerado 12 meses de evolución como criterio operativo. Desde un punto de vista regional, los registros latinoamericanos REPANARC $^{18}$, que incluyó 173 pacientes de 6 países de nuestro continente y GLADAR ${ }^{19}$, han considerado también hasta 12 meses de evolución. No obstante, evidencia reciente proveniente del estudio francés ESPOIR y de la Clínica de Artritis Temprana de Leiden ${ }^{11}$ muestran que, al menos en lo referente a lograr la remisión libre de drogas, la ventana de oportunidad tiene un umbral de 15 semanas en Leiden y 19 semanas en ESPOIR. En correspondencia, la última actualización de las guías clínicas de AR del Colegio Americano de Reumatología ${ }^{21}$ han determinado un tiempo de 6 meses (24 semanas) para definir AR temprana y de 3 meses (12 semanas) para definir AR muy temprana ("very early rheumatoid arthritis", VERA).

Las guías enfocadas específicamente en artritis 
temprana recomiendan que el paciente que presenta compromiso de más de una articulación sea derivado al reumatólogo idealmente desde las 6 semanas del debut ${ }^{22}$. En esta primera fase de funcionamiento de la unidad, los pacientes con AR tenían en promedio 8 meses de evolución de la enfermedad al momento de la consulta de tamizaje, lo cual sigue siendo subóptimo de acuerdo a la teoría de la ventana de oportunidad y las recomendaciones actuales. También es un poco más elevado comparado con las cohortes latinoamericanas: en REPANARC la duración de los síntomas antes de la primera visita era de 4,2 meses y en GLADAR de 5,8 meses. Sin embargo, es menor si se compara con experiencias puntuales en países desarrollados, donde incluso el tiempo de latencia alcanza hasta los 11 meses $^{23}$. La presencia de criterios clínicos de derivación más específicos puede explicar el tiempo de latencia y el bajo porcentaje de pacientes con artritis indiferenciada en nuestra serie (5\%) comparado con el de las otras series mencionadas, que se acercan al $15-30 \%$, donde la simple presencia de artralgia/artritis menor a 1 año basta para incluir al paciente dentro de una unidad temprana de artritis.

Efectivamente, los criterios clínicos de derivación definidos para nuestra UART fueron más detallados que los que se han utilzado en las cohortes de artritis temprana mencionadas, ya que solo comprenden la presencia de artralgia menor a un año. Por una parte, la elaboración de los criterios obedeció a una fase inicial del programa en la cual era importante recibir un número abordable de pacientes. Esto permitió seleccionar individuos con alto riesgo de poseer una $\mathrm{AR} y$ así cumplir con plazos de derivación aceptables, evitando prolongar los tiempos de atención hasta convertir a la UART en un policlínico regular de la especialidad. De hecho, a pesar de la capacitación realizada, casi un tercio de los pacientes derivados a nuestra unidad tenían más de 1 año de evolución de los síntomas. Por otra parte, la implementación de los criterios clínicos permitió seleccionar aquellos de mejor rendimiento y concordancia entre el médico general y el reumatólogo. La rigidez matinal representó el criterio más sensible con una concordancia alta y, la sinovitis, fue el criterio más específico con una concordancia moderada, destacando ambas dentro del grupo de herramientas clínicas utilizadas para derivar pacientes con sospecha de AR.
Respecto a la cohorte de 43 pacientes con AR temprana, recogemos en esta publicación solo los datos clínicos basales. Por una parte, es destacable que ningún paciente presentara daño óseo erosivo al momento del diagnóstico. Desde el punto de vista terapéutico, es destacable el tiempo de inicio del FARME en los primeros 30 días desde la consulta de tamizaje ${ }^{24}$. Será de gran interés en el seguimiento de la cohorte, los resultados clínicos referentes a inducción de remisión, daño erosivo y funcional, tasa de respuesta a FARMEs y tasa de remisión libre de drogas. Adicionalmente, y dada la alta incidencia de HTA, DM2 y dislipidemia, un punto importante serán los resultados en la morbi-mortalidad cardiovascular.

En la actualidad, la UART no existe en nuestro país de forma institucionalizada. Si los resultados con una estrategia terapéuica convencional ajustada en el tiempo demuestran un beneficio significativo en el seguimiento prospectivo de los pacientes con AR temprana, creemos que ello contribuirá a fomentar la creación y diseminación de este tipo de unidades en otros lugares de Chile.

Agradecimientos: Los autores desean agradecer a los doctores Fernando Figueroa e Yves-Marie Pers por sus contribuciones, sugerencias y aportes en orden a mejorar la calidad del presente artículo.

\section{Referencias}

1. Gibofsky A. Epidemiology, pathophysiology, and diagnosis of rheumatoid arthritis: A Synopsis. Am J Manag Care 2014; 20 (S7): S128-35.

2. Informe Final estudio de carga de enfermedad y carga atribuible, Chile 2007. Departamento de Salud Pública, Escuela de Medicina, Pontificia Universidad Católica de Chile Jul 2008. Disponible en: www.cienciasdelasalud-udla.cl/.../Informe-final-carga-Enf-2007.pdf [Consultado el 2 de enero de 2017].

3. Tiippana-Kinnunen T, Paimela L, Peltomaa R, Kautiainen $\mathrm{H}$, Laasonen L, Leirisalo-Repo M. Work disability in Finnish patients with rheumatoid arthritis: a 15-year follow-up. Clin Exp Rheumatol 2014; 32 (1): 88-94.

4. Smolen J, Landewe R, Breedveld F, Dougados M, Emery $\mathrm{P}$, Gaujoux-Viala C, et al. EULAR recommendations for the management of rheumatoid arthritis with synthetic and biological disease-modifying antirheumatic drugs. Ann Rheum Dis 2010; 69: 964-75.

5. Gremese E, Salaffi F, Bosello S, Ciapetti A, BobbioPallavicini F, Caporali R, et al. Very early rheumatoid 
arthritis as a predictor of remission: a multicentre real life prospective study. Ann Rheum Dis 2013; 72: 858-62.

6. Finckh A, Liang MH, van Herckenrode CM, de Pablo P. Long-term impact of early treatment on radiographic progression in rheumatoid arthritis: A meta-analysis. Arthritis Rheum 2006; 55 (6): 864-72.

7. Mottonen T, Hannonen P, Korpela M, Nissila M, Kautiainen $\mathrm{H}$, Ilonen J, et al. Delay to institution of therapy and induction of remission using single-drug or combination-disease-modifying antirheumatic drug therapy in early rheumatoid arthritis. Arthritis Rheum 2002; 46: 894-8.

8. van Nies J, Krabben A, Schoones J, Huizinga T, Kloppenburg M, van der Helm-van Mil A. What is the evidence for the presence of a therapeutic window of opportunity in rheumatoid arthritis? A systematic literature review. Ann Rheum Dis 2014; 73: 861-70.

9. van der Kooij S, Goekoop-Ruiterman Y, de Vries-Bouwstra J, Guler-Yuksel M, Zwinderman A, Kerstens $\mathrm{P}$, et al. Drug-free remission, functioning and radiographic damage after 4 years of response-driven treatment in patients with recent-onset rheumatoid arthritis. Ann Rheum Dis 2009; 68: 914-21.

10. Schipper L, Vermeer M, Kuper H, Hoekstra M, Haagsma C, Den Broeder A, et al. A tight control treatment strategy aiming for remission in early rheumatoid arthritis is more effective tan usual care treatment in daily clinical practice: a study of two cohorts in the Dutch Rheumatoid Arthritis Monitoring registry. Ann Rheum Dis 2012; 71: 845-50.

11. van Nies J, Tsonaka R, Gaujoux-Viala C, Fautrel B, van der Helm-van Mil A. Evaluating relationships between symptom duration and persistence of rheumatoid arthritis: does a window of opportunity exist? Results on the Leiden early arthritis clinic and ESPOIR cohorts. Ann Rheum Dis 2015; 74: 806-12.

12. Vermeer M, Kievit W, Kuper H, Braakman-Jansen L, Bernelot Moens H, Zijlstra T, et al. Treating to the target of remission in early rheumatoid arthritis is cost-effective: results of the DREAM registry. BMC Musculoskelet Disord 2013; 14: 350.

13. Goekoop-Ruiterman Y, de Vries-Bouwstra J, Allaart C, van Zeben D, Kerstens P, Hazes J, et al. Clinical and radiographic outcomes of four different treatment strategies in patients with early rheumatoid arthritis (the BeSt study): a randomized, controlled trial. Arthritis Rheum 2005; 52: 3381-90.

14. Moreland LW, O'Dell JR, Paulus HE, Curtis JR, Bathon JM, St Clair EW, et al. A randomized comparative effectiveness study of oral triple therapy versus etanercept plus methotrexate in early aggressive rheumatoid arthritis: the treatment of Early Aggressive Rheumatoid Arthritis Trial. Arthritis Rheum 2012; 64 (9): 2824-35.

15. Sokka T, Pincus T. An early rheumatoid arthritis treatment evaluation registry (ERATER) in the United States. Clin Exp Rheumatol 2005; 23: S178-81.

16. Bridges SL Jr, Hughes LB, Mikuls TR, Howard G, Tiwari HK, Alarcón GS, et al. Early rheumatoid arthritis in African-Americans: the CLEAR Registry. Clin Exp Rheumatol 2003; 21: S138-45.

17. Sokka T, Willoughby J, Yazici Y, Pincus T. Databases of patients with early rheumatoid arthritis in the USA. Clin Exp Rheumatol 2003; 21: S146-53.

18. Ramagli A, Corbacho I, Linhares F, de Abreu P, Teijeiro R, Garau M, et al. Characteristics of patients with early-onset arthritis in latin america: description of the REPANARC cohort. J Clin Rheumatol 2015; 21 (6): 283-8.

19. Cardiel MH, Pons-Estel BA, Sacnun MP, Wojdyla D, Saurit V, Marcos JC, et al. Treatment of early rheumatoid arthritis in a multinational inception cohort of latin american patients: the GLADAR experience. J Clin Rheumatol 2012; 18 (7): 327-35.

20. van Nies JA, Tsonaka R, Gaujoux-Viala C, Fautrel B, van der Helm-van Mil AH. Evaluating relationships between symptom duration and persistence of rheumatoid arthritis: does a window of opportunity exist? Results on the Leiden early arthritis clinic and ESPOIR cohorts. Ann Rheum Dis 2015; 74 (5): 806-12.

21. Smolen JS, Landewé R, Bijlsma J, Burmester G, Chatzidionysiou K, Dougados M, et al. EULAR recommendations for the management of rheumatoid arthritis with synthetic and biological disease-modifying antirheumatic drugs: 2016 update. Ann Rheum Dis 2017; doi: 10.1136 [Epub ahead of print].

22. Combe B, Landewe R, Daien CI, Hua C, Aletaha D, Álvaro-Gracia JM, et al. 2016 update of the EULAR recommendations for the management of early arthritis. Ann Rheum Dis 2016; doi: 10.1136 [Epub ahead of print].

23. Corominas H, Narváez J, Díaz-Torné C, Salvador G, Gómez-Caballero ME, de la Fuente D, et al. Diagnostic and therapeutic delay of rheumatoid arthritis and its relationship with health care devices in Catalonia. The AUDIT study. Reumatol Clin 2016; 12 (3): 146-50.

24. Verstappen SMM, Jacobs JWG, van der Veen MJ, Heurkens AH, Schenk Y, ter Borg EJ, et al. Intensive treatment with methotrexate in early rheumatoid arthritis: aiming for remission. Computer assisted management in early rheumatoid arthritis (CAMERA, an open-label strategy trial). Ann Rheum Dis 2007; 66: 1443-9. 